\title{
A Miniaturized Spectrometer Radiometer Based on MMIC Technology for Tropospheric Water Vapor Profiling
}

\author{
Flavio Iturbide-Sanchez, Student Member, IEEE, Steven C. Reising, Senior Member, IEEE, \\ and Sharmila Padmanabhan, Student Member, IEEE
}

\begin{abstract}
The fabrication of a miniaturized ground-based water vapor profiling radiometer demonstrates the capability of monolithic microwave and millimeter-wave integrated circuit technology to reduce the mass and volume of microwave remote sensing instrumentation and to reduce substantially the necessary operational power consumption and size of the radiofrequency and intermediate-frequency sections. Since those sections comprise much of the mass and volume of current microwave receivers, the fabrication of this system represents an important contribution to the design of microwave radiometers. This miniaturized radiometer implementation is particularly well suited to benefit from the cost savings associated with mass production. The small size of the radiometer $(24 \times 18 \times 16 \mathrm{~cm})$ reduces the power required by the temperature control system and allows a rapid warm-up to the temperature set point as well as maintenance of a highly stable internal temperature. Exhibiting very similar statistical properties, the four channels of the radiometer have measured Allan times of greater than $40 \mathrm{~s}$. Measurement results demonstrate that the instrument achieves a sensitivity of better than $0.2 \mathrm{~K}$ for $3 \mathrm{~s}$ of integration time. Preliminary comparisons of measured brightness temperatures with simulation results based on radiosonde data show good agreement, which are consistent with previously reported results.
\end{abstract}

Index Terms-Atmospheric measurements, humidity measurement, microwave radiometry, monolithic microwave and millimeter-wave integrated circuits (MMICs), multichip modules (MCMs), remote sensing.

\section{INTRODUCTION}

A PPLICATIONS of ground-based microwave radiometers for Earth remote sensing include the measurement of meteorological parameters such as water vapor, temperature, and cloud liquid water, which are critical for weather prediction and climate monitoring. Typically, remote sensing measurements are performed during field campaigns, due to the substantial cost and effort required for sensor deployment.

Manuscript received June 20, 2006; revised March 10, 2007. This work was supported by the National Science Foundation under Grant ATM-0456270 to Colorado State University and Grant ATM-0239722 to the University of Massachusetts Amherst.

F. Iturbide-Sanchez was with the Microwave Remote Sensing Laboratory, University of Massachusetts, Amherst, MA 01003 USA. He is now with the Microwave Systems Laboratory, Colorado State University, Fort Collins, CO 80523-1373 USA (e-mail: fiturbid@ecs.umass.edu).

S. C. Reising and S. Padmanabhan are with the Department of Electrical and Computer Engineering, Colorado State University, Fort Collins, CO 805231373 USA (e-mail: Steven.Reising@ColoState.edu).

Color versions of one or more of the figures in this paper are available online at http://ieeexplore.ieee.org.

Digital Object Identifier 10.1109/TGRS.2007.898444
Therefore, these observations are mostly limited by the availability of microwave sensors and resources to carry out the campaigns.

Due to their ability to measure with high temporal resolution and in nearly all weather conditions, a number of researchgrade and commercially available ground-based microwave radiometers are used to profile meteorological parameters, four examples of which are described here. First, the All-Sky Multiwavelength Radiometer, which is a research-grade radiometer, was developed by the University of Bern, Bern, Switzerland, for remote sensing of tropospheric water vapor, temperature, and cloud liquid water in real time [1]. Second, deployed in Alaska in 2004 by the U.S. National Oceanic and Atmospheric Administration, the 25-channel Ground-based Scanning Radiometer (GSR) is used to profile tropospheric humidity and temperature. The higher frequency channels of the GSR (183 GHz and above) are particularly well suited to the low amounts of water vapor in Arctic regions [2]. Third, a commercially available radiometer, Radiometrics Corporation's TP/WVP-3000, retrieves temperature and humidity profiles from measurements at four channels on the upper sideband of the $22-\mathrm{GHz}$ water vapor absorption line, one channel near $30 \mathrm{GHz}$, and seven channels in the $50-60-\mathrm{GHz}$ band [3]. It measures each frequency channel sequentially with an architecture that features frequency agility to enhance immunity to radio-frequency interference. Fourth, the commercially available RPG-HATPRO is a temperature and humidity profiling radiometer built by Radiometer Physics $\mathrm{GmbH}$, with six channels on the upper sideband of the $22-\mathrm{GHz}$ water vapor absorption line, one near $30 \mathrm{GHz}$, and seven channels in the 50-60-GHz band. This instrument also exploits the advantages of simultaneous direct detection of many channels to enable boundary layer scanning over a short time interval [4].

Networks of relatively small, lightweight, and low-power microwave remote sensors performing coordinated continuous measurements of the atmosphere have the potential to provide substantial improvement in spatial and temporal sampling of key thermodynamic variables in the atmosphere [5]. In the longer term, such measurements may provide data for 3-D variational assimilation (3DVAR) into numerical weather prediction (NWP) models at much higher spatial and temporal resolutions than are currently available, motivating improvement in NWP models through improved initialization and validation. Implementation of such remote sensing networks to cover significant areas necessitates substantial reduction in the unit 
cost of the microwave instrumentation. Additionally, simple and reliable system architectures will be required to facilitate the fabrication and maintenance of large numbers of microwave sensors.

The fabrication of microwave ground-based radiometers for remote sensing applications is typically implemented utilizing waveguide and connectorized components. In particular, waveguide components have dimensions inversely proportional to the operating frequency, making them heavy and bulky at low frequencies. At higher frequencies, particularly well into the millimeter and submillimeter wave ranges, waveguide components become costly and difficult to manufacture.

In recent years, the wireless communication and defense industries have invested substantial effort in the design of microwave components with low supply voltages and current consumption to reduce the power budget of microwave systems. Additionally, microwave designers have focused on the development of compact and portable systems. These efforts have led to the maturation of monolithic microwave and millimeter-wave integrated circuit (MMIC) technology, which is characterized by compact components, with a typical chip area of $1-10 \mathrm{~mm}^{2}$ and a chip thickness of only $0.1 \mathrm{~mm}$ [6]. MMICs are most often based on gallium arsenide (GaAs) technology. However, advances in electron-beam lithography and rapid development of devices based on alloys and heterostructures such as silicon germanium (SiGe), aluminum-gallium arsenide (AlGaAs), and indium-gallium arsenide (InGaAs)/GaAs have enabled the fabrication of higher frequency, lower noise, and more powerefficient MMIC components, as compared to less expensive technologies [7].

Consumer demand for direct broadcast television satellites, cellular telephony, and other telecommunication products has transformed a predominantly military-driven technology into a competitive commercial one [8]. Currently, MMICs extend in frequency range up to at least $100 \mathrm{GHz}$ [9]. Because they are produced in large quantities, commercial MMICs have typical retail prices of tens of USD per unit, in contrast to thousands of USD per unit typical of connectorized components. There is an ample variety of commercially available MMICs, including amplifiers, switches, noise sources, attenuators, mixers, frequency multipliers, oscillators, phase shifters, and power detectors. MMIC manufacturers are now developing millimeterwave commercial components for a variety of applications [10]. Due to their shorter wavelengths, further research is expected to enable the fabrication of ever smaller and lighter receivers and transmit/receive modules.

At present, the use of low-cost commercial MMICs in the design of remote sensing instruments is growing in popularity. Development of a new generation of microwave sensors based on this technology creates a need for system designers with expertise in remote sensing as well as in MMIC performance, handling, packaging, and integration. Through a strategic collaboration between the Laboratory for Millimeter-wave Devices and Applications at the University of Massachusetts (UMass) Amherst and the Microwave Systems Laboratory at Colorado State University, a new miniaturized spectrometer radiometer has been fabricated. The compact microwave radiometer for humidity profiling (CMR-H) is a small, lightweight, and highly stable prototype that consumes little power.
The CMR-H was designed, in part, to facilitate the transition of ground-based remote sensing of water vapor in the troposphere from research to operational use.

Previously, a single-channel microwave radiometer was used to measure the atmospheric emission at a number of elevation angles to retrieve the 2-D structure of the absorption coefficient at $23.8 \mathrm{GHz}$, near the $22.235-\mathrm{GHz}$ water vapor absorption line [11]. This work could be extended to a network of radiometers to retrieve vertical profiles of water vapor as a function of latitude and longitude. This requires the coordinated operation of many microwave "sensor nodes" in a network. The CMR-H was designed for network operation, in which each sensor node performs a complete volumetric scan within 5-10 min, and measurements from multiple sensor nodes are combined tomographically to retrieve vertical water vapor profiles both at and between sensor node locations. In addition to water vapor profiling radiometers such as the CMR-H, temperature profilers, and cloud liquid water, channels would be needed for remote measurement of a full suite of meteorological parameters.

\section{MMiC TeChNOLOGY IN THE DESIGN OF MiCROWAVE RADIOMETERS}

MMIC technology has demonstrated high reliability and reduced mass, volume, cost, and power consumption as compared to conventional microwave technologies [12]-[14], which are commonly used in remote sensing instrumentation. Several examples of the use of MMIC technology in the fabrication of microwave radiometers for spaceborne applications are presented in this section.

\section{A. JASON Microwave Radiometer (JMR)}

The JMR onboard the JASON-1 radar altimetry satellite measures atmospheric refractivity variations due to water vapor to provide corrections to the apparent radar range. JMR has three radiometer channels at 18.7, 23.8, and $34 \mathrm{GHz}$ [15]. Reduction of the size and weight of JMR without increases in the cost and complexity was achieved by using MMICs and hybrid microwave integrated circuits. The receiver at the primary water vapor channel, which is $23.8 \mathrm{GHz}$, was fabricated using indium phosphide (InP) MMIC technology [16]. This Dicke radiometer module, including all components up to the voltageto-frequency converter, is only $13 \times 6 \times 4 \mathrm{~cm}$ and consumes less than $2 \mathrm{~W}$ of power. JMR has a noise figure of $4.5 \mathrm{~dB}$, giving a radiometric sensitivity of better than $0.14 \mathrm{~K}$ for a 1 -s integration time.

\section{B. Integrated Multispectral Atmospheric Sounder (IMAS)}

The IMAS program was initiated as a second-generation atmospheric sounder for precision atmospheric temperature measurements from space. As part of this program, a $118-\mathrm{GHz}$ MMIC-based total power radiometer module was developed, with dimensions of $8.2 \times 6 \times 3 \mathrm{~cm}$, a power consumption of about $2.5 \mathrm{~W}$, and a mass of about $0.3 \mathrm{~kg}$ [13], [16]. The use of low-noise InP MMIC technology in its front end gave a radiometric sensitivity of about $0.6 \mathrm{~K}$ for an integration time of $1 \mathrm{~s}$. 
TABLE I

MMIC-BASEd RADIOMETER MOdULES

\begin{tabular}{|c|c|c|c|}
\hline Parameter & $\begin{array}{c}\text { JMR - 23.8 GHz } \\
{[13],[16]}\end{array}$ & $\begin{array}{c}\text { IMAS - 118 GHz } \\
{[13],[16]}\end{array}$ & $\begin{array}{c}\text { HSWBM }^{1} \\
{[17]}\end{array}$ \\
\hline Mass (kg) & $<=0.50$ & 0.30 & 0.037 \\
\hline DC power $(\mathrm{W})$ & $<2.10$ & 2.70 & $0.08-0.13$ \\
\hline $\begin{array}{l}\text { Dimensions }(\mathrm{m}) \\
\text { or Volume }\left(\mathrm{m}^{3}\right)\end{array}$ & $0.13 \times 0.06 \times 0.04$ & $0.08 \times 0.06 \times 0.03$ & $4.5 \times 10^{-6}$ \\
\hline Type & $\mathrm{DI}^{2}$ & $\mathrm{TP}^{3}$ & $\mathrm{TP}^{3}$ \\
\hline Includes & From $\mathrm{RF}$ to $\mathrm{VFC}^{4}$ & $\begin{array}{l}\text { From RF to IF } \\
\text { before detection }\end{array}$ & $\begin{array}{l}\text { From RF to } \\
\text { before A/D }\end{array}$ \\
\hline \multicolumn{4}{|c|}{$\begin{array}{l}1 \text { High-sensitivity W-band MMIC radiometer module } \\
2 \text { Dicke } \\
3 \text { Total Power } \\
4 \text { Voltage to frequency converter }\end{array}$} \\
\hline
\end{tabular}

\section{High-Sensitivity W-Band MMIC Radiometer Module (HSWMRM)}

The HSWMRM is a radiometer module designed for a focalplane array (FPA) for passive imaging of millimeter-wave thermal radiation. This module uses direct-detection architecture to reduce the size and complexity of the radiometer. With a mass of only $37 \mathrm{~g}$ and a power consumption of about $100 \mathrm{~mW}$, this radiometer module includes components from the radiofrequency (RF) section up to the analog-to-digital (A/D) converter input [17]. For similar applications in the same band and with comparable characteristics, the development of the passive millimeter-wave (PMMW) FPA camera demonstrated the usefulness of MMIC technology in the fabrication of radiometer modules in large volumes based on an automated assembly process [18].

\section{PLANCK Mission}

The PLANCK mission of the European Space Agency is the third generation of space cosmic microwave background temperature anisotropy missions [19]. The low-frequency instrument for PLANCK is a radiometer with channels at 30, 44, and $70 \mathrm{GHz}$. Due to the use of ultra-low-noise MMIC lownoise amplifiers (LNAs) based on cryogenic InP high-electron mobility transistors, very low sensitivities were achieved in differential receivers at $30 \mathrm{GHz}$ [20]. The integration of several MMIC components in multichip modules (MCMs) significantly decreased the volume of the radiometer. The power consumption of its front-end module is $27 \mathrm{~mW}$, and it has a system noise temperature of $9 \mathrm{~K}$.

Table I summarizes the characteristics of several of the radiometer modules described previously. It is important to note that these modules do not include antennas, embedded computers, data acquisition (DAQ), or temperature control subsystems.

The design of microwave radiometers based on MMIC technology has been dominated by the development of instrumentation for aerospace applications. This is motivated by the rigorous limitations imposed on the mass, volume, and power consumption of satellite payloads. As Table I confirms, this development has led to the fabrication of compact, lightweight, and power-efficient radiometer modules. An additional motivation for the use of the MMIC technology in remote sensing applications, as demonstrated in the development of the PMMW, is the potential for mass production of radiometer modules, drastically reducing their per-unit cost. However, in

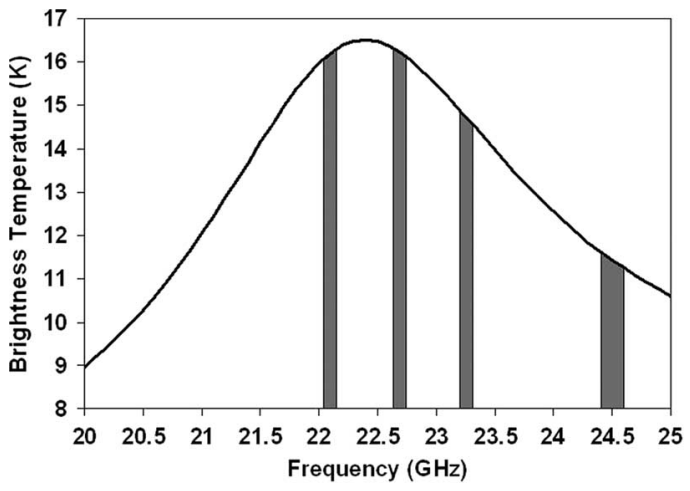

Fig. 1. Four K-band channels of the CMR-H.

the case of ground-based remote sensing applications, the use of MMIC technology is not yet widespread.

\section{CMR-H}

\section{A. System Architecture}

Collaboration among researchers with expertise in radiometry and MMIC design enabled the fabrication of the CMR-H. The CMR-H is a ground-based radiometer with four frequency channels for vertical profiling near the $22.235-\mathrm{GHz}$ water vapor absorption line. The selection of the specific frequencies and the number of channels was based on recent studies wherein the water vapor density weighting functions were calculated at a number of frequencies to determine the ensemble of frequencies near the 22.235-GHz water vapor absorption line with maximum information content for the retrieval of tropospheric water vapor profiles [21], [22]. These studies helped to eliminate frequency channels which provide no new information, since their water vapor density weighting functions are merely a linear combination of those at other frequencies. The four frequency channels selected from this study can provide linearly independent observations near the water vapor absorption line. Based on these considerations, four frequencies were chosen for the retrieval and profiling of tropospheric water vapor: 1) $22.12 \mathrm{GHz}$; 2) $22.67 \mathrm{GHz}$; 3) $23.25 \mathrm{GHz}$; and 4) $24.50 \mathrm{GHz}$. Fig. 1 shows the frequency channels of the CMR-H and their corresponding bandwidths near the $22.235-\mathrm{GHz}$ water vapor absorption line. The line shape in Fig. 1 was calculated based on recent atmospheric absorption models [23]-[26] using profiles measured by an RS-92 radiosonde in Boulder, $\mathrm{CO}$, with a total precipitable water vapor of $5.3 \mathrm{~mm}$.

The CMR-H is implemented in a superheterodyne architecture to achieve selectivity of better than $1 \%$ in bandwidth without the need for high-frequency waveguide cavity filters, as shown in Fig. 2. Except for the antenna and the local oscillator, the RF section is completely enclosed in a single MCM. Four cascaded MMIC intermediate frequency (IF) amplifiers are responsible for providing $65-70 \mathrm{~dB}$ of the power gain of the system. Following the IF amplifiers, a broadband Wilkinson power divider and a bank of four low-insertion-loss narrowband filters enable simultaneous measurements at the IFs $f_{1}=2.88 \mathrm{GHz}, f_{2}=3.43 \mathrm{GHz}, f_{3}=4.01 \mathrm{GHz}$, and $f_{4}=$ $5.25 \mathrm{GHz}$, corresponding, respectively, to the 22.12-, 22.67-, 23.25-, and 24.50-GHz K-band channels of the CMR-H. The 


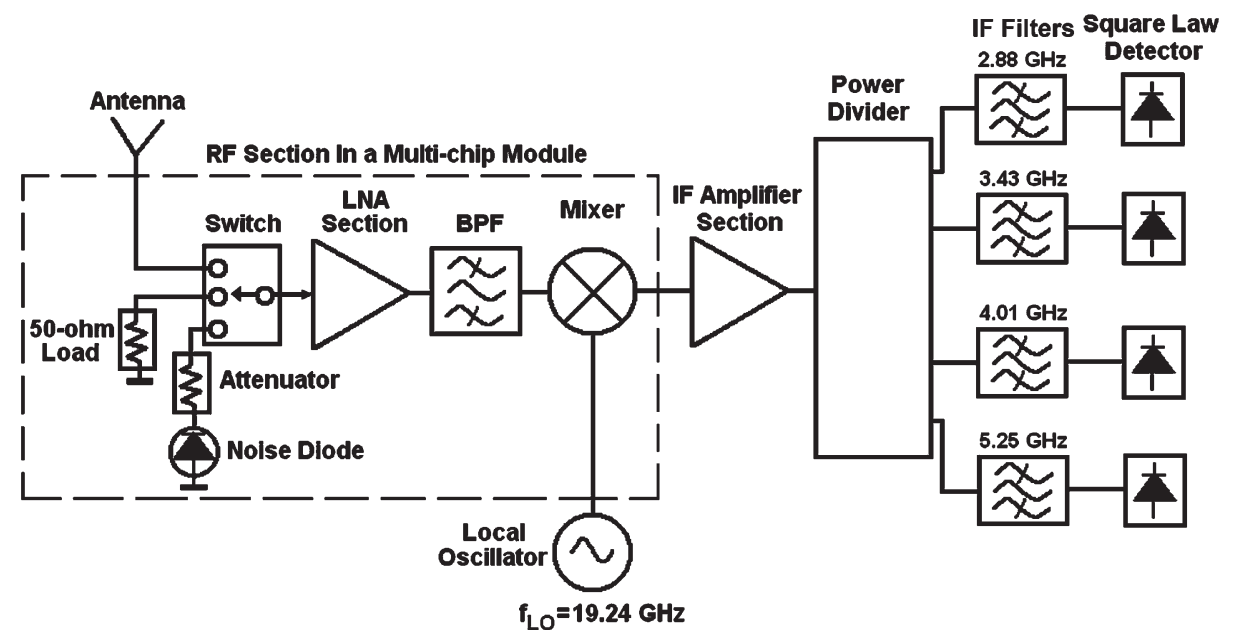

Fig. 2. Block diagram of the CMR-H system.

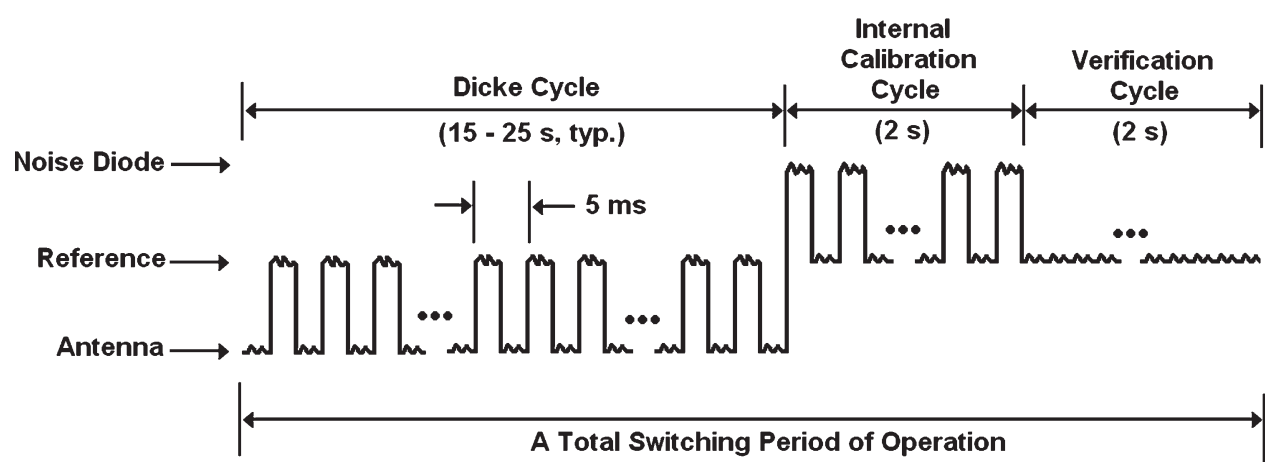

Fig. 3. Waveform showing the timing of the three different cycles of the CMR-H.

measured 3-dB bandwidths of the four IF channels are 110, 120, 120 , and $260 \mathrm{MHz}$, respectively.

To overcome short-term variations in gain and noise figure while maintaining high stability, the CMR-H uses a Dicke switch that is digitally controlled by an embedded computer and a peripheral interface controller (PIC) microcontroller. The switch alternately connects the receiver input to the antenna or to an internal ambient temperature reference implemented using a 50- $\Omega$ load. The use of a Dicke configuration, redundant noise diode injection for gain calibration, and a very stable temperature control system has been shown to result in a highly stable radiometer system [27]. In the front end of the CMR$\mathrm{H}$, a noise diode is used to perform internal calibration by means of the periodic observation of a highly stable noise diode through an attenuator (hot reference) and a 50- $\Omega$ load at ambient temperature (cold reference). Fig. 3 shows the timing of three different "cycles" that occur during the normal operation of the CMR-H. Those cycles are labeled as Dicke, internal calibration, and verification cycles. During the Dicke cycle, scene (antenna) and reference load observations alternate with $50 \%$ duty cycle every $5 \mathrm{~ms}$. The duration of the Dicke cycle is set by software to be typically $15-25 \mathrm{~s}$. The next cycle is a 2-s cycle for internal calibration, observing alternately the noise diode and the reference load every $5 \mathrm{~ms}$. During the internal calibration cycle, the noise diode output is measured relative to that of the reference load, just as the antenna temperature is measured relative to the same reference load during the Dicke cycle. Finally, a 2-s verification cycle is used to identify small $\mathrm{dc}$ offset variations in the ground of the system by observing the reference load only. For a Dicke cycle duration of $20 \mathrm{~s}$, and 2-s duration each for the internal calibration and verification cycles, the overall duty cycles of the antenna, reference load, and noise diode are $41.6 \%, 54.2 \%$, and $4.2 \%$, respectively. Each radiometer channel is sampled every $50 \mathrm{~ms}$. The samples are recorded on an embedded hard disk drive and grouped based on the cycle of the radiometer. During postprocessing, the measurements in each cycle are calibrated and averaged based on the integration time $\tau$, and erroneous measurements are removed using a quality-control algorithm.

\section{B. Antenna}

High main-beam efficiency, high radiation efficiency, narrow beamwidth, and low sidelobe levels are key requirements of antennas for passive microwave remote sensing. The corrugated conical horn antenna (or "scalar feed horn" in reflector antenna systems) is one of the most widely used antennas for passive microwave remote sensing. The directivity of a horn antenna is a function of its aperture diameter and length, so that an antenna with a larger diameter will provide higher directivity, resulting in antennas with narrower beamwidth. One way to reduce the length of such an antenna is to place an appropriate lens at the aperture of the horn. However, lenses are inherently lossy. Reflections at the dielectric interfaces can reduce not only the main-beam efficiency but also the radiation efficiency of lens antennas [28]. 


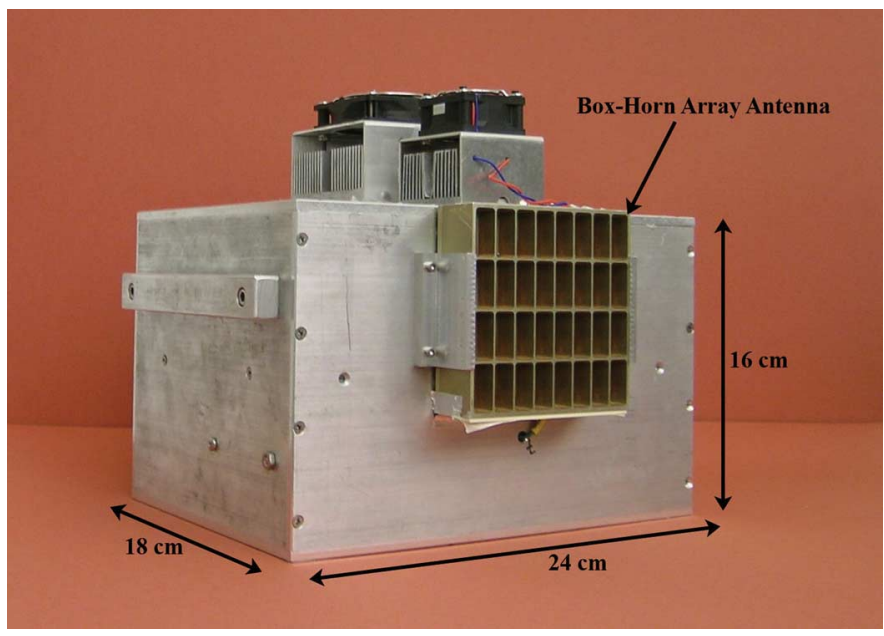

Fig. 4. CMR-H. The radome of the antenna is not shown for better visualization of the instrument.

The planarity of microstrip antennas is very attractive in the design of lightweight and compact microwave systems. Largeaperture microstrip array antennas provide high gain using very low profile structures. However, narrow bandwidth and low radiation efficiency due to radiation, ohmic, and dielectric losses in the microstrip or stripline feed networks are typical limitations of these designs [29]. One design that overcomes these limitations is a planar antenna array with a waveguide feed network; this system has quite low transmission loss, due only to the waveguide junctions, even at high frequencies.

The box-horn array (BHA) antenna is a unique quasi-planar antenna with box horns for radiating elements [30]. The BHA is characterized by its broadband waveguide feed network, which has low losses and high return loss over its bandwidth. The BHA has been demonstrated as a high-gain, small-size, and low-sidelobe antenna design [31], [32] and has been used previously for remote sensing of water vapor and cloud liquid water [33].

As shown in Fig. 4, the CMR-H uses a BHA of $4 \times 8$ waveguide elements manufactured in brass. This BHA has a bandwidth of $11 \%$ ( 22.0 to $24.6 \mathrm{GHz}$ ), single linear polarization, greater than $90 \%$ main-beam efficiency, and a return loss of $14.5,16.0,19.0$, and $12.5 \mathrm{~dB}$ at the radiometer operating frequencies of 22.12, 22.67, 23.25, and $24.50 \mathrm{GHz}$, respectively. The antenna's $3-\mathrm{dB}$ beamwidth is $7-8^{\circ}$, and its gain varies from about 25 to $27 \mathrm{dBi}$ over the radiometer's frequency range. With a mass of $360 \mathrm{~g}$, the size of the antenna is only $10.25 \times 10.25 \times$ $2.5 \mathrm{~cm}$, with a WR-34 waveguide flange as the RF port. WR-34 is a rectangular waveguide standard that bridges the $\mathrm{K}$ - and Ka-bands for operation from $22-33 \mathrm{GHz}$. A radome made of Rohacell 31-HF was installed to thermally isolate the antenna from changes in sun and wind and to provide some protection from condensation.

\section{Implementation of the RF Section in an MCM}

The advantages of MMIC technology can be enhanced by packaging and combining the functionality of individual MMIC components in MCMs, reducing a system's mass, volume, complexity, and number of lossy microstrip-to-coaxial or microstrip-to-waveguide transitions. MMIC dies/chips require

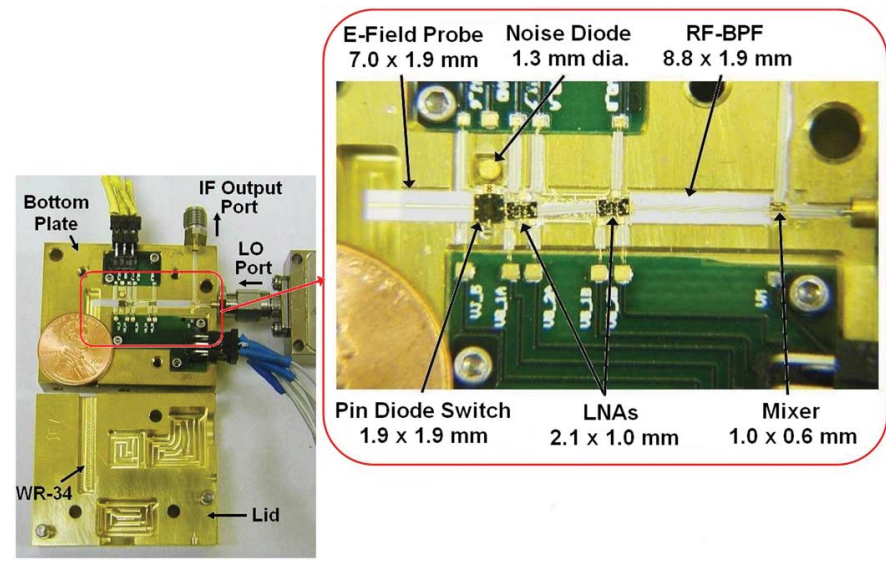

Fig. 5. (Left) Top view of the bottom and top plates and (right) detail of the bottom plate of the highly integrated RF-MCM of the CMR-H.

mechanical support as well as thermal and electrical protection. These can be provided by reliable packaging that preserves the electrical performance of the enclosed MMICs [34]. Plastic, metal, ceramic, and thin-film multilayer packages have been used in the design of MCMs [35], [36]. Due to its mechanical reliability, good thermal conductivity, electromagnetic shielding, and manufacturability, a brass package was designed for the RF-MCM of the CMR-H, with gold plating to reduce chemical oxidation, as shown in Fig. 5. This highly integrated MCM not only reduces the size of the radiometer but also improves its thermal stability since most of the RF active and passive components are housed in a small module.

Typical problems that are faced during the design of metal packages are the potential coupling among MMIC components and resonances of the waveguide cavities inherently created in metallic housings. To provide access to the MMICs during the testing and assembly stages, one of the design requirements of the RF-MCM was to have a nearly flat surface in the block where the MMICs were mounted. Most of the MMIC components were cascaded in a narrow and shallow channel ( $0.4 \mathrm{~mm}$ in depth) with a calculated $\mathrm{TE}_{10}$ cutoff frequency of about $75 \mathrm{GHz}$, which corresponds to more than three times the maximum operating frequency of the CMR-H. When designing systems operating at higher frequencies, the dimensions of the narrow channel need to be chosen carefully to avoid the propagation of undesired waveguide modes that can lead to amplifier instabilities, for example. However, MMICs inherently have small widths to facilitate packaging in narrow channels.

The RF-MCM consists of two pieces: 1) the lid and 2) the bottom plate. The MMICs, passive microwave components, and microstrip transmission lines are mounted on the bottom plate (see Fig. 5). The lid is accurately aligned to the bottom plate. It encloses the RF components and completes the housing of the RF-MCM. Compatible with the waveguide port of the BHA antenna described previously, the input port of the RF-MCM is a rectangular milled trench extending from the left side of the bottom plate shown in Fig. 5, i.e., half of a WR-34 waveguide. The other half of the waveguide was milled in the lid of the RF-MCM. The electric field lines in the walls of a rectangular waveguide excited with a $\mathrm{TE}_{10}$ mode are symmetric about the center of the broad wall [37]. Therefore, to reduce the resistive losses in the waveguide, the RF-MCM was fabricated so that 


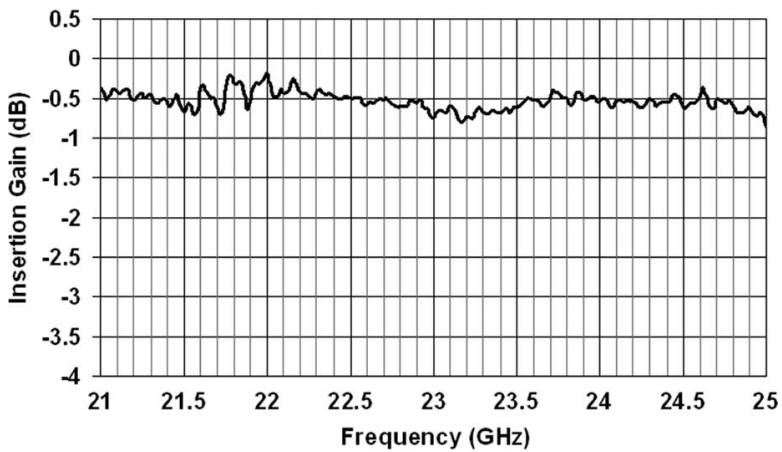

Fig. 6. Measured insertion loss of the longitudinal E-field probe.

the junction of the lid and the bottom plate is longitudinal and in the center of the broad wall.

A high-performance, broadband, compact, and reliable longitudinal E-field probe was designed [38] as a waveguide-tomicrostrip transition that efficiently couples microwave energy from the waveguide to the MMICs. As shown in Fig. 6, the E-field probe manufactured in alumina has a measured insertion loss of $0.53 \pm 0.1 \mathrm{~dB}$ over the entire frequency range of the CMR-H. As the first component in the RF-MCM, its lowinsertion loss minimizes the probe's negative effect upon the sensitivity of the radiometer system.

The LNAs of the RF-MCM are responsible for the RF amplification of the radiometer and have an important effect on the noise temperature of the system. The two LNAs are biased using a common gate voltage $\left(V_{g}\right)$ and a common drain voltage $\left(V_{d}\right) . V_{d}$ was set to a fixed value of $3.5 \mathrm{~V}$, but $V_{g}$ can be selected in software to set the required gain and noise temperature of the radiometer.

Following the LNAs is a six-section microstrip coupled line bandpass filter, with a bandwidth of $26.5 \%$, a center frequency of $23.0 \mathrm{GHz}$, an insertion loss of $1.5 \mathrm{~dB}$, and a loss flatness of $\pm 0.25 \mathrm{~dB}$

The down-conversion of the system is achieved by using a passive (no bias required) double-balanced mixer. A 19.24-GHz low-phase-noise continuous-wave signal is injected into the mixer through the local oscillator (LO) port to generate the IFs, as shown in the right panel in Fig. 5. The IF signals produced by the RF-MCM are output through the IF port.

As part of this project, two RF-MCMs were fabricated and tested to examine the variability in the gain performance of two different units, as shown in Fig. 7. Although driven with the same bias voltages, i.e., gate voltage $\left(V_{g}\right)$ and drain voltage $\left(V_{d}\right)$, the second RF-MCM (RF2) provides about 4-5 dB more gain than the first RF-MCM (RF1) at the IFs of the CMR-H, denoted as $f_{1}, f_{2}, f_{3}$, and $f_{4}$. The differences in the gain are not related to differences in the assembly or packaging; instead, they are related to the higher current consumption measured in the LNAs in RF2. Although the LNAs were fabricated at the same foundry, process variations always exist. However, as seen in Fig. 7, one can adjust the bias conditions of the RF-MCMs to optimize the system performance. Since the gain of the system is mostly driven by the high-gain IF amplifiers, the bias voltages of the RF-MCM were not set for maximum gain, but instead were optimized for minimum noise figure. With about $4.5 \mathrm{GHz}$ of bandwidth, the RF-MCM has dimensions of $4.5 \times 3.7 \times$

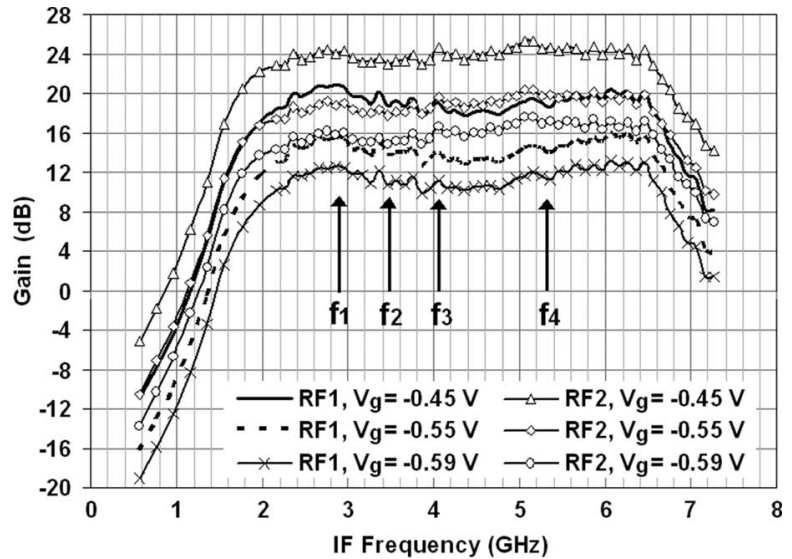

Fig. 7. Gain performance of two different RF-MCMs (RF1 and RF2) measured at the IF port under different gate bias conditions. In all cases, the drain voltage is $V_{d}=3.5 \mathrm{~V}$

$2.5 \mathrm{~cm}$, a mass of $260 \mathrm{~g}$, and a power consumption of no more than $500 \mathrm{~mW}$, comparable to the modules reported in Table I.

\section{Dicke Switch}

The preferred switch technology for microwave radiometers is ferrite switches due to their low loss. In recent years, significant investment has been made to miniaturize and reduce the mass of ferrite switches [39]. However, they are still large compared to solid-state switches compatible with MMIC designs, such as p-i-n diode and FET switches. Although p-i-n diode switches traditionally have greater insertion loss than ferrite switches, they have the advantages of higher switching speeds, higher isolation, longer life, and reduced power consumption. Commercially available MMIC p-i-n diode switches have $0.5-1.5-\mathrm{dB}$ insertion loss, isolation of $40-45 \mathrm{~dB}$, and operating frequencies up to $80 \mathrm{GHz}$. The Dicke switch of the CMR-H was implemented using a single-pole triple-throw (SP3T) p-i-n diode switch with an insertion loss of $0.8-1.5 \mathrm{~dB}$ and isolation varying from 35 to $40 \mathrm{~dB}$, as measured over the CMR-H frequency range. In contrast to ferrite switches, this switch uses small surface-mount drivers that consume only $75 \mathrm{~mW}$. The switch is controlled by a programmable logic device according to the timing pattern shown in Fig. 3.

\section{E. Noise Injection in the CMR-H}

An internal hot calibration reference implemented with a stable noise diode is integrated into the RF-MCM. This hot reference is used to provide reliable internal calibration and gain correction. The integration of the noise source into the RF-MCM offers mechanical robustness and simplifies thermal control, improving thermal stability. This noise source generates white Gaussian noise with a typical excess noise ratio of $20 \mathrm{~dB}$, an equivalent noise temperature of $29000 \mathrm{~K}$. To reduce its output noise temperature to about $500 \mathrm{~K}$, an MMIC-based attenuator was integrated between the noise diode and the $\mathrm{p}-\mathrm{i}-\mathrm{n}$ diode switch.

When the Dicke switch is set to connect the noise diode and attenuator to the first LNA and the noise diode is turned off, this provides a second ambient temperature reference 


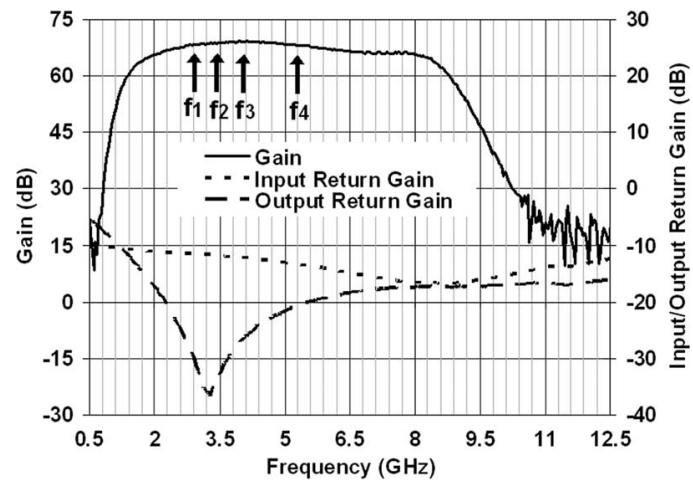

Fig. 8. Gain performance of two cascaded IF-MCMs. The vertical scale on the left shows the gain, and the vertical scale on the right gives the input and output return gains.

comparable to the 50- $\Omega$ load. Therefore, this radiometer architecture provides the option of having more than one ambient temperature reference or of replacing the 50- $\Omega$ load with another noise diode to provide a second hot temperature reference. In this regard, $\mathrm{p}-\mathrm{i}-\mathrm{n}$ diode switches with more than three switch positions would provide a larger set of temperature references for internal calibration and verification of receiver linearity.

\section{F. IF Section}

After the K-band (RF) frequencies are amplified, filtered, and mixed down to IFs in the RF-MCM, they are fed to the IF amplification section, which provides $70 \%$ of the power gain of the radiometer system. Two IF-MCMs were designed to house commercial, highly linear, high gain, and high power efficiency MMIC amplifiers. Each of these two modules has dimensions of $2.0 \times 1.25 \times 0.45 \mathrm{~cm}$, a mass of $50 \mathrm{~g}$, and consumes $600 \mathrm{~mW}$.

The IF amplifier section (two IF-MCMs) provides 65-70 dB of power gain over a frequency range of $2-8 \mathrm{GHz}$, as shown in Fig. 8. To operate in their linear region, the IF-MCMs were biased to output a maximum power of $30 \mathrm{~dB}$ below their 1-dB output compression point. In general, great care is needed in the design of the power and voltage gain stages because linear operation is critical to the absolute calibration of the radiometer.

After amplification of the IFs, the four channels of the CMR-H are selected using a Wilkinson power divider and a bank of passive filters. Since the CMR-H measures four K-band channels over a $60 \%$ IF bandwidth, a broadband two-section Wilkinson power divider was designed to provide improved bandwidth over a single-section design and high isolation among the output ports [40]. The measured performance of the power divider is shown in Fig. 9. The insertion loss varies from 6.5-7.5 dB over the IF bands of the CMR-H. The highest insertion loss is measured in the $f_{4}$ channel. The input and output return losses of the power divider are always better than $10 \mathrm{~dB}$. To minimize the size of the power divider, it was fabricated on a high permittivity substrate and using thin-film resistors.

After power division, the four channels of the CMR-H are defined by highly selective, compact, and low-loss surface acoustic wave filters packaged in surface-mount technology. To improve the isolation between the channels and provide



Fig. 9. Measured performance of the two-section coupled microstrip Wilkinson power divider. The vertical scale on the left shows the insertion gain, and the vertical scale on the right gives the input and output return gains.

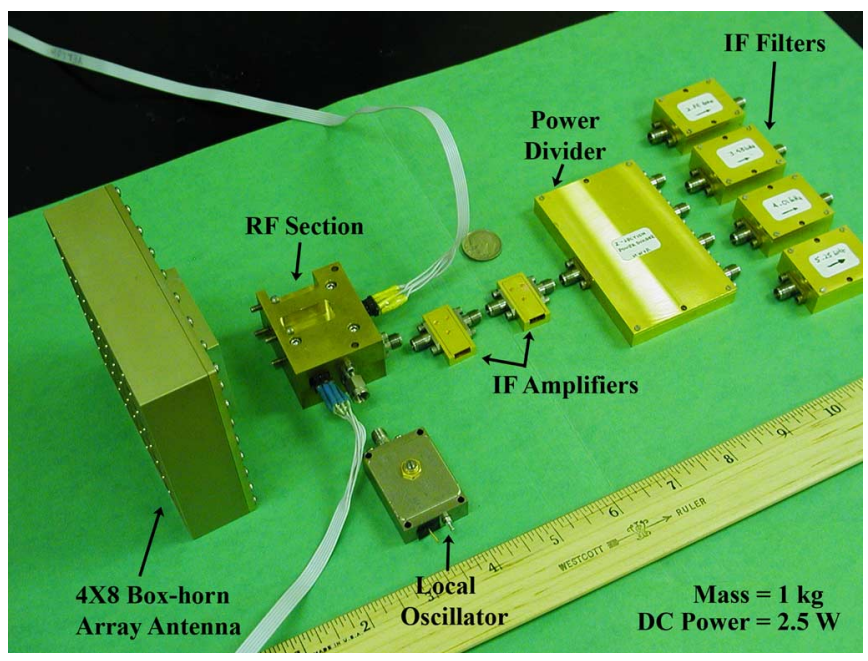

Fig. 10. Submodules of the CMR-H, including antenna, RF, and IF sections.

flexibility in the selection of the CMR-H frequencies, each filter was packaged in its own gold-plated brass housing.

Due to use of a quasi-planar array antenna and of MMIC technology in the design and implementation of the RF and IF sections of the CMR-H, the total mass and power consumption of all components from the antenna to the IF filters are only $1 \mathrm{~kg}$ and less than $2.5 \mathrm{~W}$, respectively. Fig. 10 provides a photograph of the antenna, RF, and IF modules of the CMR-H.

\section{G. Power Detection and Video Amplifier Section}

After the IF section, each signal is input to a zero-bias square law power detector that produces a voltage proportional to the microwave power at the output of the corresponding IF filter. To maintain a linear relationship between power and voltage, the detector needs to operate in its square law range. In the $\mathrm{CMR}-\mathrm{H}$, the power level at the input to the power detectors is approximately $15 \mathrm{~dB}$ below its $1-\mathrm{dB}$ compression point to keep the power detector operating in the square law region, as measured from the power detector's transfer curve.

The voltage outputs of the power detectors are amplified and conditioned for A/D conversion in the video amplifier section. Low-noise and high-gain precision operational amplifiers are used to amplify the dc voltage inputs. The video amplifier section consumes only $60 \mathrm{~mW}$. 




Fig. 11. Measured noise temperature of the CMR-H.

\section{H. $D A Q$}

The CMR-H also has an internal compact general purpose single-board computer that provides autonomous real-time data processing. The DAQ system connected to the embedded computer has $16 \mathrm{~A} / \mathrm{D}$ inputs with 12-bit resolution, 24 digital I/O lines, and four programmable analog outputs. The A/D inputs of the DAQ are organized as follows: four sample the Dicke radiometer voltages of the four RF channels; seven are connected to outputs of resistance temperature detectors sensing temperatures at different locations in the radiometer; four sense the currents drawn by the RF and IF amplifiers; and one is used to read the position of the radiometer from a clinometer. An Ethernet port on the CMR-H chassis enables remote control of the instrument and real-time assessment of the quality of measured data. This allows the radiometer to exchange data and commands with other sensor nodes in a network.

\section{Noise Temperature of the CMR-H}

Using the well-known Y-factor method [41], the noise temperature of the CMR-H was measured. Following this method, the CMR-H measured two temperature references: 1) microwave absorber at ambient temperature (hot reference) and 2) microwave absorber immersed in liquid nitrogen $\left(\mathrm{LN}_{2}\right.$, cold reference). Fig. 11 shows the measured noise temperature of the CMR-H for the RF1 MCM under three different LNA bias conditions. The noise temperatures shown in Fig. 11 were measured using a power meter and an electronically tunable filter with a $50-\mathrm{MHz}$ bandwidth. These data at the frequencies $f_{1}, f_{2}, f_{3}$, and $f_{4}$ agree with the noise temperatures measured using the CMR-H IF filters at the corresponding frequencies to within $50-100 \mathrm{~K}$. This discrepancy is due to the difference between the 50-MHz measurement bandwidth used in Fig. 11 and the larger bandwidth of the IF filters of the CMR-H. The equivalent noise temperature of the RF2 MCM is from 100 to $200 \mathrm{~K}$ larger than the one measured in the RF1 MCM under the same bias conditions. However, the equivalent noise temperature of the RF2 MCM can be lowered by using different LNA bias voltages.

When the LNAs of the RF1 MCM were driven with a gate voltage of $V_{g}=-0.45 \mathrm{~V}$, the measured results showed a good tradeoff between gain and equivalent noise temperature. Under this bias condition, the radiometer has a noise temperature between 500 and $800 \mathrm{~K}$ over the range from the lowest $\left(f_{1}\right)$ to

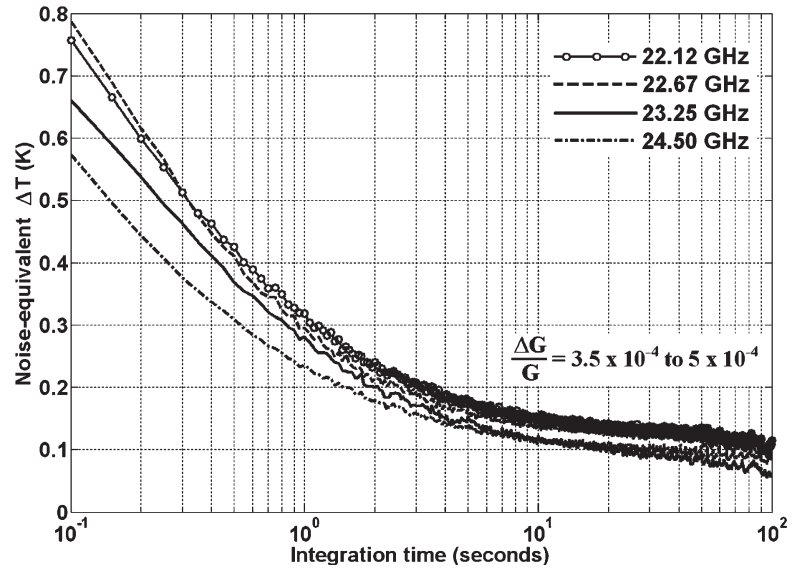

Fig. 12. Measured radiometric resolution of the four channels of the CMR-H.

the highest $\left(f_{4}\right)$ IF bands of interest. Fig. 11 shows that the noise performance of the radiometer rapidly degrades at the edges of the antenna bandwidth.

\section{J. Measurement Sensitivity of the CMR-H}

The sensitivity of a Dicke radiometer, which is often termed the "noise equivalent $\Delta T$ " $(N E \Delta T)$, is given as [42]

$$
\begin{aligned}
N E \Delta T=\left[\frac{2\left(T_{A}+T_{\mathrm{REC}}\right)^{2}+2\left(T_{\mathrm{REF}}+T_{\mathrm{REC}}\right)^{2}}{B \tau}\right. \\
\left.+\left(\frac{\Delta G_{S}}{G_{S}}\right)^{2}\left(T_{A}-T_{\mathrm{REF}}\right)^{2}\right]^{\frac{1}{2}}
\end{aligned}
$$

where $T_{A}, T_{\mathrm{REC}}$, and $T_{\mathrm{REF}}$ represent the antenna temperature, the receiver equivalent noise temperature, and the internal reference temperature, respectively. $B$ and $\tau$ represent the system bandwidth and integration time, respectively.

The measured sensitivities of the four channels of the CMR-H as a function of integration time are presented in Fig. 12. From (1), the sensitivity of a Dicke radiometer depends on the antenna temperature $T_{A}$. Since the CMR-H is designed to measure sky brightness temperatures, and the effect of receiver gain variations $(\Delta G / G)$ depends on $T_{A}-$ $T_{\mathrm{REF}}$, the sensitivity of the radiometer was measured by viewing a stable and clear sky for $30 \mathrm{~min}$. Before and after this measurement, the instrument was calibrated using external references (see Section III-M). The radiometer was not recalibrated during the 30-min period of observation. The average values of $T_{A}$ during this experiment were 34.0, $33.8,30.0$, and $22.0 \mathrm{~K}$ for the 22.12-, 22.67-, 23.25-, and 24.50-GHz frequency channels of the CMR-H. The channel with the largest bandwidth, with an RF of $24.50 \mathrm{GHz}$, corresponding to an IF of $5.25 \mathrm{GHz}$, shows the lowest (best) sensitivity values for integration times less than $10 \mathrm{~s}$. These results demonstrate that for integration times greater than $3 \mathrm{~s}$, the CMR-H achieves a radiometric resolution of better than $0.2 \mathrm{~K}$. Channels at RFs of 23.25 and $24.50 \mathrm{GHz}$, corresponding to IFs of 4.01 and $5.25 \mathrm{GHz}$, exhibit a sensitivity of better than $0.1 \mathrm{~K}$ for integration times greater than $20 \mathrm{~s}$.

The sensitivity of a radiometer measuring a stable antenna temperature $T_{A}$, with constant $T_{\mathrm{REC}}$ and $T_{\mathrm{REF}}$, could be decreased by increasing either the integration time $\tau$ or the 
radiometer channel bandwidth $B$, or by decreasing the gain variations $\Delta G / G$. In the design of a radiometer, the value of $B$ is fixed and, in some cases, may be selected to improve the sensitivity of the instrument. However, the parameter $\Delta G / G$ mostly depends on the design and properties of the radiometer, including the stability of the temperature, biasing, and component values. Therefore, increasing the integration time is a common technique to improve the $N E \Delta T$ of the radiometer. Since the integration time $\tau$ only affects the first term of (1), the sensitivity of the radiometer for long integration times will be determined by the gain variations $\Delta G / G$. Therefore, the linear decrease (on a semilog plot) in sensitivity with integration time, given by the first term of (1), will not hold for long integration times. For instance, for 10-100 s of integration time, the sensitivity of the CMR-H decreases by only about $0.05 \mathrm{~K}$ (region dominated by the gain fluctuations $\Delta G / G$ ), whereas from 0.1 to $1 \mathrm{~s}$ of integration time, the sensitivity decreases by $0.35-0.5 \mathrm{~K}$ (region dominated by dependence on $\tau$ ). Measurements of emission from very stable clear skies and the use of (1) show that the gain variations in the CMR-H $(\Delta G / G)$ vary from about $3.5 \times 10^{-4}$ to $5 \times 10^{-4}$. In addition, $\Delta G / G$ was independently determined by measuring the noise diode inside the CMR-H for about $90 \mathrm{~min}$. The $\Delta G / G$ when measuring the noise diode was $\sim 1 \times 10^{-4}$, which is comparable to the $\Delta G / G$ when measuring clear sky.

\section{K. Bias and Temperature Stability of the CMR-H}

A number of current and temperature sensors monitor the bias and temperature stability of the CMR-H. The IF-MCMs, which provide $65-70 \mathrm{~dB}$ (most) of the power amplification, exhibit a bias current stability of better than $\pm 0.04 \mathrm{~mA}$ $(0.03 \%-0.04 \%)$. In addition, the bias current stability of the RF LNAs is better than $\pm 0.09 \mathrm{~mA}(0.1 \%)$. These results show good stability in the bias system, which is important for determining the stability of the overall system, as will be explained in Section III-L.

Fig. 13 shows a cross-sectional view of the principal components of the CMR-H. It consists of two main cavities, housing the RF/IF subsystem and the digital subsystem. The first cavity contains the RF and IF components, the video amplifier board, and the power detectors. The second cavity houses the embedded computer, the electronics boards, and the temperature control system. Governed by separate control circuits, the system thermoelectric cooler (system-TEC) and the RF/IF-TEC control the temperature inside the radiometer. When the system is switched on, the system-TEC is immediately initialized to reach the programmed temperature set point of the entire system. This temperature is typically reached in less than $30 \mathrm{~min}$ for ambient external temperatures between $-5{ }^{\circ} \mathrm{C}$ and $35{ }^{\circ} \mathrm{C}$. After this temperature has been stabilized, the RF/IF-TEC is initialized to the same system temperature set point for precise temperature control of the RF-MCM, the two IF-MCMs, and the local oscillator, which are mounted on a single plate that is thermally isolated from the rest of the system. Due to the significant amount of heat dissipated by the embedded computer, fans are used to circulate the air and dissipate heat in the digital cavity.

Because of the importance of temperature control to the stability of the radiometer's gain and noise figure, and to the

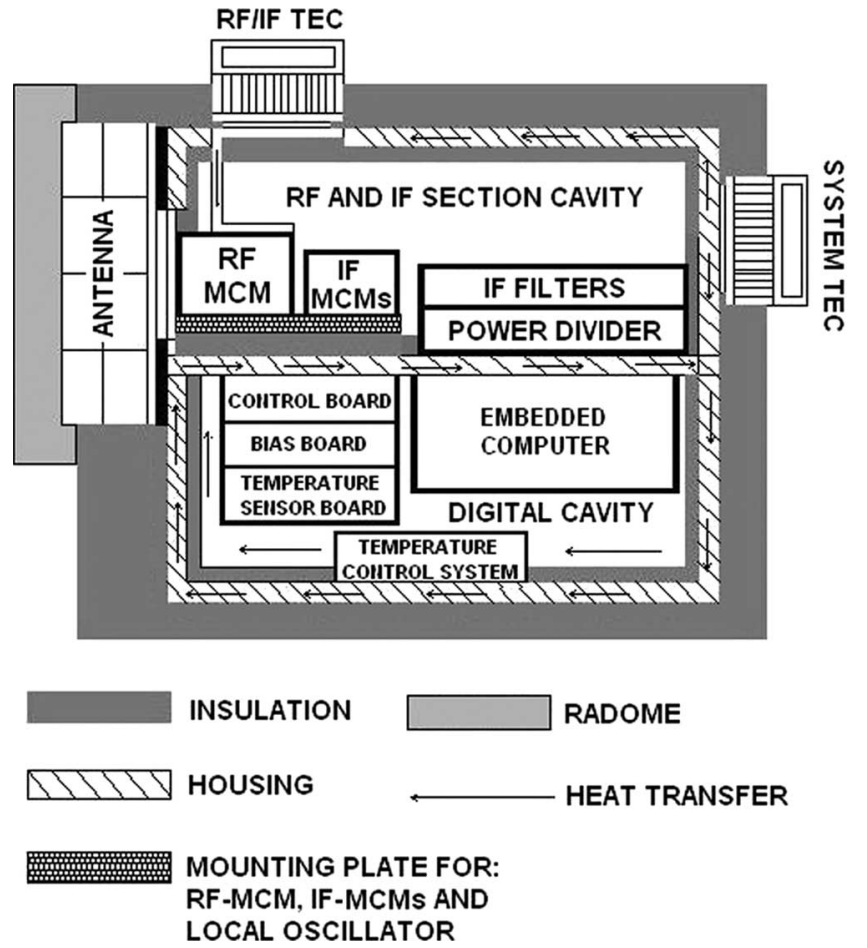

Fig. 13. Illustration of the functional and thermal control subsystems of the CMR-H.

stability of the hot and ambient internal temperature references, two temperature sensors were mounted inside the RF-MCM. During operation in the field environment, temperature measurements show that the RF-MCM has a stable temperature, with a maximum variability of $0.1 \mathrm{~K}$. When ambient temperature or strong winds change quickly, the temperature variability of the system may increase, but the temperature control system recovers within $5 \mathrm{~min}$ and reduces the variability to $0.1 \mathrm{~K}$ or less. A very large fraction of the total power consumption of the CMR-H is due to the temperature control system. The maximum total power consumption is about $50 \mathrm{~W}$ when operating the instrument at ambient external temperatures from $-5{ }^{\circ} \mathrm{C}$ to $35^{\circ} \mathrm{C}$. Of this total, $16 \mathrm{~W}$ is consumed by the internal embedded computer, the DAQ system, and the hard disk drive.

\section{Measurement Stability of the CMR-H}

The stability of microwave receivers is fundamentally limited by instabilities in electronic components, power supply voltages, and thermodynamic temperatures. Allan variance has been used to characterize the stability of microwave instrumentation because it can help to identify and better understand the noise mechanisms limiting stability [43]-[45]. The Allan variance is calculated from time series of the calibrated antenna temperatures measured by the radiometer and sampled every $t$ seconds. A total of $N$ measured antenna temperatures are divided into $M$ groups of $k$ measurements each. The average value of each group is given as

$$
T_{i}(k)=\frac{1}{k} \sum_{n=1}^{k} T_{B(i k+n)}, \quad i=0, \ldots, M
$$




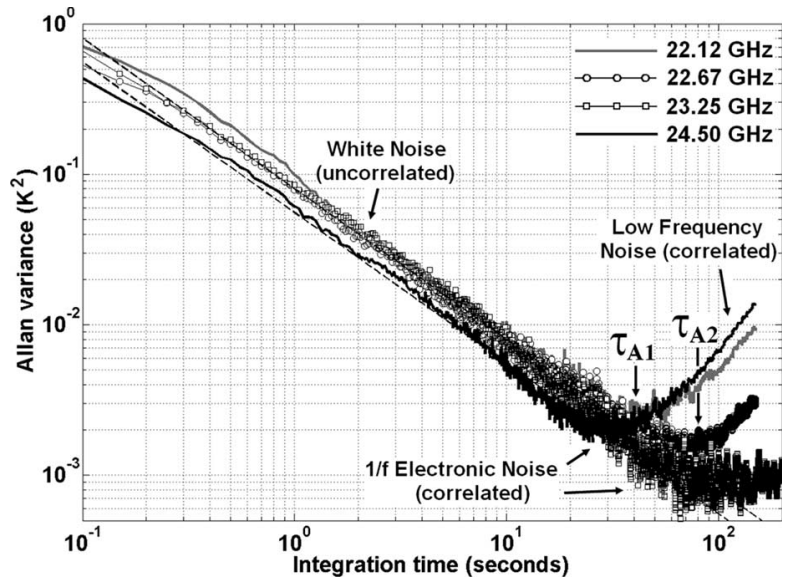

Fig. 14. Measured Allan variance for the four channels of the CMR-H, when observing a stable microwave absorber at ambient temperature.

The Allan variance is half the mean-square difference between each set of two contiguous averages $T_{i}, T_{i+1}$ and is calculated as

$$
\sigma_{A}^{2}(k)=\frac{1}{2 M} \sum_{i=1}^{M-1}\left(T_{i+1}(k)-T_{i}(k)\right)^{2} .
$$

The Allan variance was obtained by measuring the brightness temperature $\left(T_{B}\right)$ of a stable microwave absorber at ambient temperature $(300 \mathrm{~K})$ for $2 \mathrm{~h}$ and by measuring clear and stable sky for $30 \mathrm{~min}$. For the case of clear-sky observation, we measured an average value of $T_{A}$ equal to $48.7,46.5,41.9$, and $32.4 \mathrm{~K}$ for the 22.12-, 22.67-, 23.25-, and 24.50-GHz frequency channels of the CMR-H. Before and after this set of measurements, the instrument was calibrated using external references (see Section III-M). The calibration parameters of the radiometer obtained from these two measurements were compared for validation purposes. The radiometer was not recalibrated during the measurements of microwave absorber or those of clear sky. The measured variability in the thermodynamic temperature of the microwave absorber during the 2-h Allan variance measurement was $\pm 0.02 \mathrm{~K}$. The variability of the measured clear-sky brightness temperatures was \pm 1.07 , $\pm 1.15, \pm 1.24$, and $\pm 1.01 \mathrm{~K}$ for the 22.12-, 22.67-, 23.25-, and 24.50-GHz channels of the CMR-H, respectively. The Allan variance for the four channels of the CMR-H when observing a stable microwave absorber at ambient temperature is plotted in Fig. 14 as a function of integration time $\tau=k t$. The two dashed lines with a slope of -1 show the Allan variance plot that would result from measuring purely uncorrelated (white) noise.

Three principal regions are identified on the Allan variance plot. The white noise region follows the first term of (1), with a slope of -1 . A second region, the $1 / f$-noise region, is characterized by a zero slope. This region is the result of flicker or $1 / f$ electronic noise in the electronics. Finally, low-frequency drift noise in the electronics is responsible for an Allan variance with a slope greater than or equal to one. The integration time at which the Allan variance deviates from the first term of the radiometer equation is known as the Allan time $\tau_{A}$. According to this definition and based on Fig. 14, the 22.12- and 24.50-GHz channels have a measured $\tau_{A}$ of $\tau_{A 1}=40 \mathrm{~s}$. Integration times

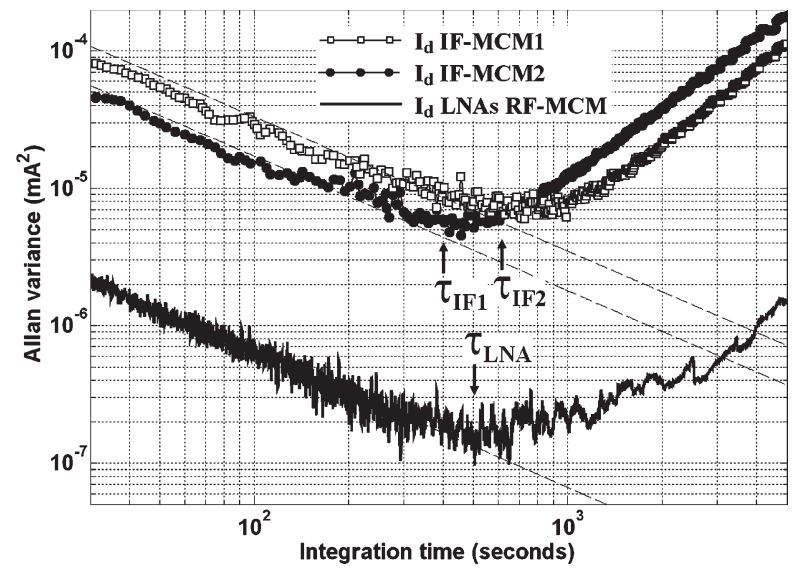

Fig. 15. Measured Allan variance of the bias current of the main power amplification components of the CMR-H.

longer than $40 \mathrm{~s}$ do not lower the noise level of those two channels. The 22.67- and 23.25-GHz channels have a measured $\tau_{A}$ of $\tau_{A 2}=80 \mathrm{~s}$, so it is possible to obtain the expected sensitivity benefit up to integration times of $80 \mathrm{~s}$. For the case of the clear-sky observations, the measured Allan time $\tau_{A}$ is $20 \mathrm{~s}$ at the four channels of the CMR-H. This reduction in Allan time is related to the increase of the effect of the gain variations of the system due to the larger difference between $T_{A}$ and $T_{\mathrm{REF}}$ when measuring sky brightness temperatures, from the second term of (1).

The stability of the bias system was investigated to determine its effect on Allan times of the radiometer's antenna temperatures. Since the bias currents were sampled during both measurements, the Allan variance was calculated for the bias currents of amplifiers in both the RF-MCM and the IF-MCMs, which are the main power amplification components of the CMR-H. The Allan variance shown in Fig. 15 demonstrates that the bias system is stable up to approximately $500 \mathrm{~s}$, more than five times the Allan time of the radiometer's antenna temperatures. Therefore, the results in Fig. 14 indicate that the stability of the system is limited by $1 / f$ electronic ("flicker") noise in the electronics more than by any low-frequency drifts in the bias system.

\section{Calibration of the CMR-H}

A proper external calibration procedure is essential for accurate conversion of sampled output voltages into measured brightness temperatures. Calibration consists of observing two or more scenes with well-known brightness temperatures. For the calibration of the CMR-H, the cosmic microwave background temperature and a microwave absorber at ambient temperature were used as the two reference scenes. In the frequency range of 22-25 GHz, zenith clear-sky brightness temperatures $T_{B}$ 's measured at ground level in midlatitudes are typically a few tens of Kelvin. For those low temperature values, the tipping-curve calibration technique provides a highly accurate external reference [46]. To perform tipping curves, the CMR-H was mounted on a small positioner $(20 \times 26 \times 26 \mathrm{~cm})$ capable of precise positioning $\left(0.01^{\circ}\right)$ over ranges of $180^{\circ}$ and $360^{\circ}$ in elevation and azimuth angle, respectively. Under clear-sky 
conditions, elevation angle scans were performed, and measured $T_{B}$ 's were extrapolated to the cosmic background temperature of $2.7 \mathrm{~K}$, analogous to an effective radiometer looking upward at the top of the atmosphere. The tipping-curve calibration consists of measurements at each of six elevation angles with opacities of $1,1.25,1.5,1.75,2.0$, and 2.25 air masses. After an external calibration procedure is performed, the values of the gain and the offset are determined for linear conversion of the output voltages to measured brightness temperatures. In general, several successive calibrations are performed to determine the variability of the gain and offset values.

From sets of external calibrations using the tipping-curve technique and measurements of microwave absorber at ambient temperature, each performed within $30 \mathrm{~min}$, and by accumulating statistics during 18 days of clear-sky observations over a three-month period, we analyze the precision of the radiometer by quantifying the errors due to the variability in the calibration constants of the radiometer. Those errors are approximately $\pm 0.5 \mathrm{~K}$ for the $22.67-\mathrm{GHz}$ channel and $\pm 0.4 \mathrm{~K}$ for the $22.12-$, 23.25-, and $24.50-\mathrm{GHz}$ channels of the radiometer, at a sky brightness temperature of about $35 \mathrm{~K}$, using an integration time of $3 \mathrm{~s}$. In addition, for a brightness temperature of approximately $298 \mathrm{~K}$, the errors are about $\pm 0.25 \mathrm{~K}$ for the $22.67-\mathrm{GHz}$ channel and $\pm 0.2 \mathrm{~K}$ for the 22.12-, 23.25-, and 24.50-GHz channels, respectively, using an integration time of $3 \mathrm{~s}$.

The equivalent noise temperature of the noise diode was determined using external calibration. Based on the external calibrations used to determine the precision of the radiometer, the variability of its internal hot reference is $\pm 0.2 \mathrm{~K}$ for the 23.25-GHz channel and $\pm 0.3 \mathrm{~K}$ for the 22.12-, 22.67-, and 24.50-GHz channels, respectively, using an integration time of $3 \mathrm{~s}$. This hot reference and the $50-\Omega$ load at ambient temperature are used as two additional brightness temperature references to perform internal calibration when no external calibration is available and to correct for changes in system gain and noise figure between external calibrations.

For verification of the external calibration, observations of liquid nitrogen $\left(\mathrm{LN}_{2}\right)$ were performed. During these observations, a microwave pyramidal absorber with a maximum reflection coefficient at normal incidence of $-50 \mathrm{~dB}$ was completely immersed in $\mathrm{LN}_{2}$. The brightness temperature of the absorber was measured for 1-2 min. The need to avoid condensation or ice on the radome of the antenna limited not only the time duration but potentially also the accuracy of the $\mathrm{LN}_{2}$ observations. The agreement of the $\mathrm{LN}_{2}$ absorber measurements with the expected value was $\pm 0.9, \pm 0.7, \pm 0.8$, and $\pm 0.7 \mathrm{~K}$, for the 22.12-, 22.67-, 23.25-, and 24.50-GHz channels, respectively.

\section{N. Preliminary Measurements Using the CMR-H}

During the third week of March 2006, measurements were performed at the National Center for Atmospheric Research, Boulder, $\mathrm{CO}$, for preliminary comparison of brightness temperatures measured by the CMR-H with simulated brightness temperatures based on profiles measured by colocated and simultaneous Vaisala RS-92 radiosonde launches. This radiosonde measures temperature, humidity, and pressure with accuracies of $0.5{ }^{\circ} \mathrm{C}, 5 \%$ relative humidity $(\mathrm{RH})$, and $1 \mathrm{hPa}$, respectively [47]. A recent study found that the RH accuracy of

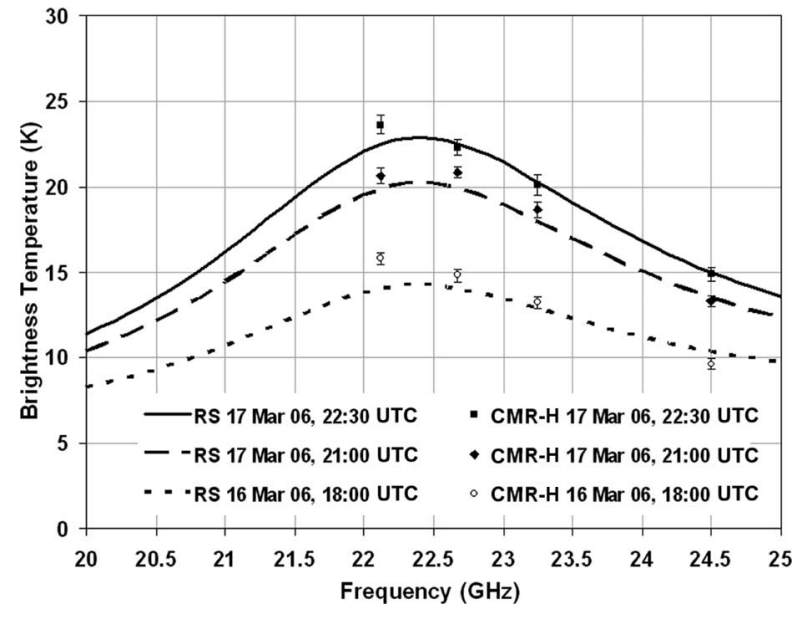

Fig. 16. Comparison between calculated $T_{B}$ 's from the water vapor absorption model based on [23]-[26] and measured $T_{B}$ 's in the four channels of the CMR-H. The times shown in the plot are UTC.

RS-92 radiosondes is $<5 \%$ in lower troposphere, and $<10 \%$ in the middle troposphere and upper troposphere [48].

Under clear-sky and cloud-free conditions, the measured $T_{B}$ 's (using an integration time of $3 \mathrm{~s}$ ) in the four K-band channels of the CMR-H were compared to calculated $T_{B}$ 's based on the most up-to-date water vapor absorption models reported in [23]-[26]. This comparison is shown in Fig. 16, showing that the largest differences between the measured $T_{B}$ 's and simulated $T_{B}$ 's from radiosonde data occur in the 22.12-GHz channel, i.e., 0.65-1.75 K. However, differences of only $0.06-0.70 \mathrm{~K}$ are observed in the $24.50-\mathrm{GHz}$ channel. The $T_{B}$ differences in the $22.67-$ and $23.25-\mathrm{GHz}$ channels are $0.75-0.85$ and $0.35-0.65 \mathrm{~K}$, respectively. These results are consistent with comparisons made by Liljegren et al. [26], who found similar differences between measured brightness temperatures and those modeled from extensive radiosonde observations. The differences were substantially larger at frequencies closer to the $22.235-\mathrm{GHz}$ water vapor absorption line. Additionally, there is an inherent error caused by the different measurement durations of radiometer and radiosonde measurements since the radiosondes reach an altitude of $12 \mathrm{~km}$ (below which lies almost all of the water vapor in the atmosphere) only after 45-60 min. Error bars were included for the measured $T_{B}$ 's, representing the standard deviation of the CMR-H measurements during the comparison period. These values vary from 0.4 to $0.6 \mathrm{~K}$, corresponding to about twice the sensitivity of the radiometer for an integration time of $3 \mathrm{~s}$ and demonstrating the stability of the sky during the 1 -h radiometer observations.

To consider additional sources of error leading to the observed differences between the measured and modeled $T_{B}$ 's, the accuracy of the humidity sensor of the RS-92 was analyzed. For that purpose, the modeled brightness temperatures were recalculated, considering the manufacturer's published uncertainty in the measured relative humidity $(5 \%)$. This analysis was performed for the three cases presented in Fig. 16. For the observation performed at 22:30 coordinated universal time (UTC) on March 17, the modeled $T_{B}$ 's had a standard deviation of 1.6, 1.5, 1.4, and $0.9 \mathrm{~K}$, at 22.12, 22.67, 23.25, and $24.50 \mathrm{GHz}$, respectively. As expected, due to their higher 
TABLE II

SPECIFICATIONS OF THE CMR-H

\begin{tabular}{|c|c|c|}
\hline Parameter & Units & Specifications \\
\hline Type of Radiometer & & Dicke, Super-heterodyne, Single Side Band \\
\hline K-band Channels & $\mathrm{GHz}$ & $22.12,22.67,23.25$ and 24.50 \\
\hline IF Channels & $\mathrm{GHz}$ & $2.88,3.43,4.01$ and 5.25 \\
\hline 3-dB Antenna Beamwidth & $\circ$ & $7-8$ \\
\hline Local Oscillator Frequency & $\mathrm{GHz}$ & 19.24 \\
\hline $\begin{array}{l}\text { Radiometer Equivalent Noise } \\
\text { Temperature }\end{array}$ & $\mathrm{K}$ & $550-800$ \\
\hline Sensitivity@1-s integration time & $\mathrm{K}$ & $0.2-0.3$ \\
\hline $\begin{array}{l}\text { Sensitivity to Precipitable Water } \\
\text { Vapor@3-s integration time }\end{array}$ & $\mathrm{mm}$ & $0.1-0.15$ \\
\hline Stability (Allan time) & $\mathrm{s}$ & $40-80$ \\
\hline Dimensions of the RF-MCM & $\mathrm{cm}$ & $4.5 \times 3.7 \times 2.5$ \\
\hline Mass of RF-MCM & $\mathrm{kg}$ & 0.26 \\
\hline $\begin{array}{l}\text { DC Power Consumption of the RF- } \\
\text { MCM }\end{array}$ & $\mathrm{mW}$ & 500 \\
\hline $\begin{array}{l}\text { Dimensions of the CMR-H (from RF } \\
\text { to digitally sampled output, } \\
\text { including embedded computer) }\end{array}$ & $\mathrm{m}$ & $0.24 \times 0.18 \times 0.16$ \\
\hline Mass of the CMR-H & $\mathrm{kg}$ & 6 \\
\hline $\begin{array}{l}\text { DC Power Consumption of the } \\
\text { CMR-H (no positioner and no dew } \\
\text { blower) }\end{array}$ & W & 50 max. $\left(-5\right.$ to $\left.35^{\circ} \mathrm{C}\right)$ \\
\hline Calibration & & $\begin{array}{l}\text { External: Tipping curve, microwave } \\
\text { absorber at ambient temperature and } \mathrm{LN}_{2} \text {. } \\
\text { Internal: Noise diode and reference load. }\end{array}$ \\
\hline
\end{tabular}

sensitivity to water vapor, the channels closest to the water vapor absorption line had the largest standard deviations, consistent with our measurements. Considering all three cases in Fig. 16, their standard deviations due to measurement uncertainty of RS-92 relative humidity varied from 1.3 to $1.6,1.3$ to $1.5,1.1$ to 1.4 , and 0.7 to $0.8 \mathrm{~K}$ at $22.12,22.67,23.25$, and $24.50 \mathrm{GHz}$, respectively.

\section{SUMmary}

The fabrication and operation of the CMR-H have demonstrated a significant reduction in mass, volume, and power consumption of the RF and IF sections of the radiometer as compared to conventional ground-based microwave radiometers, due to their implementation using highly integrated MCMs based on MMIC technology. The CMR-H has a mass of $6 \mathrm{~kg}$, a volume of $0.007 \mathrm{~m}^{3}$, and consumes a maximum of $50 \mathrm{~W}$ of power. The positioner is not included in these specifications. The small size of the radiometer reduces the power required by the temperature control system and allows a rapid warmup to the temperature set point as well as maintenance of a highly stable internal temperature. In addition, the small size of the RF- and IF-MCMs simplifies thermal control and increases thermal stability of the radiometer components with large thermal coefficients.

MMIC-based microwave systems avoid the use of many costly discretely packaged components. Due to the simple topology of the CMR-H and the high level of integration of its RF and IF sections, the parts' cost per radiometer decreases markedly when more than one unit is fabricated. Moreover, once the performance of the instrument has been verified, mass production of the principal subsystems of the radiometer can be performed using a reliable semiautomated assembly process. These results facilitate the implementation of an operational network of MMIC-based microwave radiometers in a variety of frequency bands to measure vertical profiles of meteorological variables as a function of latitude and longitude. It is expected that in the near future, in addition to individual components, highly integrated, reliable, and inexpensive modular subsystems based on MMICs will be commercially available.

The specifications of the CMR-H are summarized in Table II. Considering that the CMR-H is a complete radiometer, its performance levels are similar to previous radiometer modules based on MMIC technology fabricated for aerospace and defense applications, as presented in Section II and Table I. The size reduction achieved by using MMIC technology is complementary to direct-sampling digital radiometers [49], in which the digital section begins closer to the antenna by substituting analog RF and IF components with equivalent sampling and digital signal processing.

\section{ACKNOWLEDGMENT}

The authors would like to thank the assistance and support of Prof. R. W. Jackson and J. Wielgus of UMass Amherst during the initial phases of the CMR-H design and fabrication. Generous assistance and support during the radiosonde comparison experiment were provided by Dr. J. Vivekanandan, Dr. T. Weckwerth, Dr. J. Wang, and K. Young of NCAR, Boulder, CO. The authors would also like to thank five anonymous reviewers for their helpful comments to improve this paper. 


\section{REFERENCES}

[1] L. Martin, M. Schneebeli, and C. Mätzler, "ASMUWARA, a ground-based radiometer system for tropospheric monitoring," Meteorol. Z., vol. 15, no. 1, pp. 11-17, Feb. 2006.

[2] E. R. Westwater, M. Klein, V. Leuski, A. J. Gasiewski, T. Uttal, D. A. Hazen, D. Cimini, V. Mattioli, B. L. Weber, S. Dowlatshahi, J. A. Shaw, J. S. Liljegren, B. M. Lesht, and B. D. Zak, "Initial results from the 2004 North Slope of Alaska Arctic Winter Radiometric Experiment," in Proc. IEEE Geosci. Remote Sens. Symp., 2004, vol. 2, pp. 1374-1377.

[3] R. Ware, R. Carpenter, J. Güldner, J. Liljegren, T. Nehrkorn, F. Solheim, and F. Vandenberghe, "A multi-channel radiometric profiler of temperature, humidity and cloud liquid," Radio Sci., vol. 38, no. 4, 8079, Jul./Aug. 2003.

[4] T. Rose, S. Crewell, U. Löhnert, and C. Simmer, "A network-suitable microwave radiometer for operational monitoring of the cloudy atmosphere," Atmos. Res.-Special issue: CLIWA-NET: Observation and Modelling of Liquid Water Clouds, vol. 75, no. 3, pp. 183-200, 2005. DOI:10.1016/j.atmosres.2004.12.005.

[5] A. E. MacDonald, "A global profiling system for improved weather and climate prediction," Bull. Amer. Meteorol. Soc., vol. 86, no. 12, pp. 1747-1764, Dec. 2005.

[6] I. D. Robertson and S. Lucyszyn, RFIC and MMIC Design and Technology. London, U.K.: Inst. Electr. Eng., 2001, pp. 1-25.

[7] M. W. Pospieszalski, "Extremely low-noise amplification with cryogenic FETs and HFETs: 1970-2004," IEEE Microw. Mag., vol. 6, no. 3, pp. 62-75, Sep. 2005

[8] G. Bechtel, "GaAs: The wireless technology of the future (and the past)," in Proc. GaAs IC Symp., 2003, pp. 11-12.

[9] S. Weinreb, T. Gaier, R. Lai, M. Barsky, Y. C. Leong, and L. Samoska, "High-gain 150-215-GHz MMIC amplifier with integral waveguide transitions," IEEE Microw. Guided Wave Lett., vol. 9, no. 7, pp. 282-284, Jul. 1999.

[10] J. Powell and D. Bannister, "Business prospects for commercial mm-wave MMICs," IEEE Microw. Mag., vol. 6, no. 4, pp. 34-43, Dec. 2005.

[11] A. V. Bosisio and G. Drufuca, "Retrieval of two-dimensional absorption coefficient structure from a scanning radiometer at $23.8 \mathrm{GHz}$," Radio Sci., vol. 38, no. 3, 8038, Feb. 2003. DOI:10.1029/2002RS002628.

[12] R. Gupta, A. Zaghloul, F. Fassal, and C. Mahle, "The impact of miniaturized RF technologies on payload architectures of future digital communications satellites," in Proc. 10th Int. Conf. Digital Satellite Commun., 1995, pp. 637-645.

[13] R. W. Haas, "MMIC based MMW radiometers for remote sensing," in Proc. Int. Microw. Symp. Dig., 1999, pp. 399-402.

[14] K. Nishikawa, K. Kamogawa, B. Piernas, M. Tokumitsu, S. Sugitani, I. Toyoda, and K. Araki, "Three-dimensional MMIC technology for lowcost millimeter-wave MMICs," IEEE J. Solid-State Circuits, vol. 36, no. 9, pp. 1351-1359, Sep. 2001.

[15] S. Brown, C. Ruf, S. Keihm, and A. Kitiyakara, "Preliminary validation and performance of the JASON microwave radiometer," in Proc. IEEE Geosci. Remote Sens. Symp., 2003, vol. 2, pp. 1077-1079.

[16] M. Sholley, G. Barber, R. Raja, and C. Jackson, "Advanced MMIC radiometers," in Proc. Microw. Conf. Asia Pacific, 2000, pp. 94-98.

[17] B. Kane, S. Weinreb, E. Fischer, and N. Byer, "High-sensitivity W-band MMIC radiometer modules," in Proc. IEEE Microw. and Millimeter-Wave Monolithic Circuits Symp., 1995, pp. 15-16.

[18] G. S. Dow, D. C. W. Lo, Y. Guo, E. W. Lin, T. T. Chung, M. D. Biedenbender, O. Miromontes, A. Marashi, L. Yujiri, P. S. C. Lee, M. M. Shoucri, and B. R. Allen, "Large scale W-band focal plane array for passive radiometric imaging," in Proc. IEEE MTT-S Int. Microw. Symp. Dig., 1996, vol. 1, pp. 369-372.

[19] J. A. Tauber, "The Planck mission," Adv. Space Res., vol. 34, no. 3, pp. 491-496, 2004.

[20] B. Aja, E. Artal, L. de la Fuente, J. P. Pascual, A. Mediavilla, N. Roddis, D. Kettle, W. F. Winder, L. P. Cara, and P. de Paco, "Very low-noise differential radiometer at $30 \mathrm{GHz}$ for the PLANCK LFI," IEEE Trans. Microw. Theory Tech., vol. 53, pt. 2, no. 6, pp. 2050-2062, Jun. 2005.

[21] T. M. Scheve and C. T. Swift, "Profiling atmospheric water vapor with a K-band spectral radiometer," IEEE Trans. Geosci. Remote Sens., vol. 37, no. 3, part 2, pp. 1719-1729, May 1999.

[22] F. Solheim, J. R. Godwin, E. R. Westwater, Y. Han, S. J. Keihm, K. Marsh, and R. Ware, "Radiometric profiling of temperature, water vapor and cloud liquid water using various inversion methods," Radio Sci., vol. 33, no. 2, pp. 393-404, Mar./Apr. 1998

[23] P. W. Rosenkranz, "Water vapor continuum absorption: A comparison of measurements and models," Radio Sci., vol. 33, no. 4, pp. 919-928, Jul./Aug. 1998.
[24] P. W. Rosenkranz, "Erratum: 'Water vapor microwave continuum absorption: A comparison of measurements and models'," Radio Sci., vol. 34, no. 4, 1025, Jul./Aug. 1999.

[25] M. Y. Tretyakov, V. V. Parshin, M. A. Koshelev, V. N. Shanin, S. E. Myasnikova, and A. F. Krupnov, "Studies of $183 \mathrm{GHz}$ water line: Broadening and shifting by air, $\mathrm{N}_{2}$ and $\mathrm{O}_{2}$ and integral intensity measurements," J. Mol. Spectrosc., vol. 218, no. 2, pp. 239-245, Apr. 2003.

[26] J. C. Liljegren, S.-A. Boukabara, K. Cady-Pereira, and S. A. Clough, "The effect of the half-width of the 22-GHz water vapor line on retrievals of temperature and water vapor profiles with a 12-channel microwave radiometer," IEEE Trans. Geosci. Remote Sens., vol. 43, no. 5, pp. 1102-1108, May 2005.

[27] A. B. Tanner and A. L. Riley, "Design and performance of a high-stability water vapor radiometer," Radio Sci., vol. 38, no. 3, 8050, pp. 1-12, Mar. 2003.

[28] C. A. Balanis, Antenna Theory, Analysis and Design. New York: Harper \& Row, 1982, pp. 646-654.

[29] E. Levine, G. Malamud, S. Shtrikman, and D. Treves, "A study of microstrip array antennas with the feed network," IEEE Trans. Antennas Propag., vol. 37, no. 4, pp. 426-434, Apr. 1989.

[30] S. Silver, Microwave Antenna Theory and Design. London, U.K.: Peregrinus, 1984 , pp. 377-380.

[31] T. Sehm, A. Lehto, and A. V. Raisanen, "A large planar 39-GHz antenna array of waveguide-fed horns," IEEE Trans. Antennas Propag., vol. 46, no. 8, pp. 1189-1193, Aug. 1998.

[32] T. Sehm, A. Lehto, and A. V. Raisanen, "A high-gain 58-GHz boxhorn array antenna with suppressed grating lobes," IEEE Trans. Antennas Propag., vol. 47, no. 7, pp. 1125-1130, Jul. 1999.

[33] M. R. Agen, "Radiometric water vapor and liquid measurement near 22 GHz," M.S. thesis, Univ. Massachusetts, Amherst, 2002.

[34] T. A. Midford, J. J. Wooldridge, and R. L. Sturdivant, "The evolution of packages for monolithic microwave and millimeter-wave circuits," IEEE Trans. Antennas Propag., vol. 43, no. 9, pp. 983-991, Sep. 1995.

[35] C. A. Harper, Electronic Packaging and Interconnection Handbook. New York: McGraw-Hill, 1991, pp. 7-1-7-112.

[36] D. S. Wein, "Advanced ceramic packaging for microwave and millimeter wave applications," IEEE Trans. Antennas Propag., vol. 43, no. 9, pp. 940-948, Sep. 1995.

[37] R. E. Collin and F. J. Zucker, Antenna Theory. New York: McGraw-Hill, 1969, ch. 14, pt. I, pp. 584-587.

[38] Y. C. Leong and S. Weinreb, "Full band waveguide-to-microstrip probe transitions," in Proc. IEEE MTT-S Int. Microw. Symp. Dig., Anaheim, CA, 1999, pp. 1435-1438.

[39] EMS Technologies Inc., "Miniaturized ferrite switch networks," Microw. J., vol. 46, p. 98, Jul. 2003.

[40] S. B. Cohn, "A class of broadband three-port TEM-mode hybrids," IEEE Trans. Microw. Theory Tech., vol. MTT-19, no. 2, pp. 110-116, Feb. 1968.

[41] M. Golio, The RF and Microwave Handbook. Boca Raton, FL: Rockwell Collins, Inc., 2000, pp. 4-26-4-28.

[42] F. T. Ulaby, R. K. Moore, and A. K. Fung, Microwave Remote Sensing: Active and Passive, vol. 1. London, U.K.: Addison-Wesley, 1981, p. 372.

[43] D. W. Allan, "Statistics of atomic frequency standards," Proc. IEEE, vol. 54, no. 2, pp. 221-230, Feb. 1966.

[44] J. A. Barnes, A. R. Chi, L. S. Cutler, D. J. Healey, D. B. Leeson, T. E. McGunigal, J. A. Mullen, W. L. Smith, R. L. Sydnor, R. F. C. Vessot, and G. M. R. Winkler, "Characterization of frequency stability," IEEE Trans. Instrum. Meas., vol. IM-20, no. 2, pp. 105-120, May 1971.

[45] T. Berg, S. Cherednichenko, V. Drakinskiy, P. Khosropanah, H. Merkel, E. Kollberg, and J. W. Kooi, "Stability of HEB receivers at $\mathrm{THz}$ frequencies," Proc. SPIE, vol. 5498, pp. 605-615, 2004.

[46] Y. Han and E. R. Westwater, "Analysis and improvement of tipping calibration for ground-based microwave radiometers," IEEE Trans. Geosci. Remote Sens., vol. 38, no. 3, pp. 1260-1277, May 2000.

[47] Vaisala Radiosonde RS92-SGP, Ref. B210358EN-C, Vaisala, 2006. [Online]. Available: http://www.vaisala.com/weather/products/ soundingequipment/radiosondes/rs92/vaisala radiosonde rs92-sgp brochure.pdf

[48] L. M. Miloshevich, H. Vömel, D. N. Whiteman, B. M. Lesht, F. J. Schmidlin, and F. Russo, "Absolute accuracy of water vapor measurements from six operational radiosonde types launched during AWEX-G and implications for AIRS validation," J. Geophys. Res., vol. 111, no. D9, D09S10, Apr. 2006. DOI:10.1029/2005JD006083.

[49] M. A. Fischman and A. W. England, "An L-band direct sampling digital radiometer for STAR technology sensors," in Proc. Aerosp. Conf., 2000, vol. 5, pp. 129-139. 


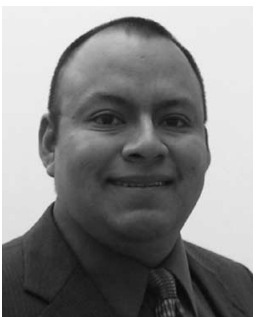

Flavio Iturbide-Sanchez (S'03) received the B.S.E.E (magna cum laude) degree in electronics engineering from Autonomous Metropolitan University, Mexico City, Mexico, in 1999 and the M.S.E.E. (summa cum laude) degree in electrical engineering from Advanced Studies and Research Center to the National Polytechnic Institute, Mexico City, in 2001. He is currently working toward the $\mathrm{Ph} . \mathrm{D}$. degree at the University of Massachusetts, Amherst. His master's research was focused on the design, development, and characterization of low-noise, low-power consumption, and highly linear RF amplifiers for personal communication systems based on silicon germanium technology.

From September 2001 to March 2005, he was a Research Assistant in the Department of Electrical and Computer Engineering, University of Massachusetts, where he performed research on the design, development, and characterization of highly integrated multichip modules and microwave circuits for low-noise, low-power consumption, high-gain, and high-stability microwave radiometers at the Microwave Remote Sensing Laboratory, University of Massachusetts. Since April 2005, he has been with the Microwave Systems Laboratory, Colorado State University, Fort Collins, as a Research Associate II, focusing on the miniaturization of microwave radiometers for remote sensing applications and the design, testing, deployment, and data analysis of the compact microwave radiometer for humidity profiling. His research interests include communication systems, microwave radiometry, microwave/millimeter-wave IC design and packaging, RF integrated circuits, system-on-a-chip, active antennas, and the modeling, analysis, design, and measurement of microwave and millimeter-wave circuits and systems.

Mr. Iturbide-Sanchez was a finalist in two IEEE Student Paper Competitions, one at the International Geoscience and Remote Sensing Symposium in Anchorage, AK, September 2004 and recently at the International Microwave Symposium in San Francisco, CA, June 2006. He was also awarded the Mexican National Council for Science and Technology (CONACYT) Graduate Fellowship from 1999 to 2004.

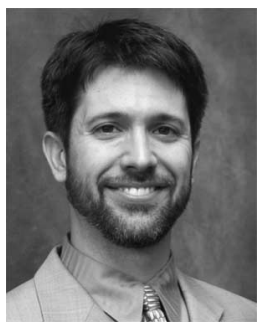

Steven C. Reising (S'88-M'98-SM'04) received the B.S.E.E. (magna cum laude) and M.S.E.E. degrees in electrical engineering from Washington University, St. Louis, MO, and the Ph.D. degree from Stanford University, Stanford, CA, in 1998, where he was advised by Prof. Umran S. Inan and supported by a NASA Earth Systems Science Fellowship. At Stanford University, his research focused on lowfrequency remote sensing of lightning and its energetic coupling to the ionosphere, which produces chemical changes and transient optical emissions.

From 1998 to 2004, he was an Assistant Professor in electrical and computer engineering at the University of Massachusetts, Amherst. During the summers of 1999, 2000, and 2003, he was a Summer Faculty Fellow in the Remote Sensing Division of the Naval Research Laboratory, Washington, DC. In September 2004, he became an Associate Professor in electrical and computer engineering at Colorado State University, Fort Collins. He has served as a reviewer for Radio Science, Geophysical Research Letters, and the Journal of Oceanography. His research interests include passive microwave and millimeter-wave remote sensing of the oceans, atmosphere, and land, including microwave radiometer systems and lidar systems for sensing of temperature and winds in the middle and upper atmosphere.

Dr. Reising serves as an Associate Editor of the IEEE GEOSCIENCE AND Remote SENSing LetTers (2004 to present) and as a Guest Editor of the Special Issue on Microwave Radiometry and Remote Sensing Applications of the IEEE Transactions on Geoscience and Remote Sensing
(TGARS), July 2007. He is an Elected Member of the IEEE Geoscience and Remote Sensing Society's (GRSS) Administrative Committee (2003-2008), where he is currently the Membership Chair. He served as the Editor of the IEEE GRSS NEWSLETTER from April 2000 to March 2003. He was the Chair of the Springfield, MA, Joint Chapter of the IEEE Antennas and Propagation Society, Geoscience and Remote Sensing Society, Electron Devices Society, Microwave Theory and Techniques Society, and Lasers and Electro-Optics Society (1999-2004) and has been a member of the Technical Program Committee of every IGARSS from 2001 to present. He is also a member of the American Meteorological Society, the American Geophysical Union, Tau Beta $\mathrm{Pi}$, and Eta Kappa Nu. He chaired the 9th Specialist Meeting on Microwave Radiometry and Remote Sensing Applications, MicroRad '06, which was held from February 28 to March 3, 2006, in San Juan, Puerto Rico. He chairs the annual United States National Committee (USNC)/International Union of Radio Science (URSI) Student Paper Competition in Boulder, CO (2004 to present). He is a member of URSI Commissions F, G, and $\mathrm{H}$ and is the Secretary of USNC Commission F (2006-2008). He has served as a reviewer for the IEEE GEOSCIENCE AND REMote SENSING LetTERS and TGARS. He received the NSF CAREER Award (2003-2008) and the Office of Naval Research Young Investigator Program (YIP) Award (2000-2003). He also received the Barbara H. and Joseph I. Goldstein Outstanding Junior Faculty Award in 2004, the Lilly Teaching Fellowship for 2001-2002, and the Young Scientist Award at the URSI General Assembly in Toronto, Canada, in 1999. While at Stanford University, he received the First Place in the USNC/URSI Student Paper Competition at the 1998 National Radio Science Meeting in Boulder.



Sharmila Padmanabhan ( $\left.\mathrm{S}^{\prime} 01\right)$ received the B.Eng. degree in electronics and telecommunications engineering from the University of Mumbai, Mumbai, India, in 2001 and the M.S. degree in electrical and computer engineering from the University of Massachusetts (UMass), Amherst, in 2004. She is currently working toward the Ph.D. degree at Colorado State University, Fort Collins. Her master's research focused on design, testing, calibration, and data analysis for a K-band polarimetric radiometer at the Microwave Remote Sensing Laboratory, UMass.

She received the Young Scientist Award at the URSI General Assembly in New Delhi, India, in 2005. While at UMass, she received the Second Place in the IEEE Geoscience and Remote Sensing Student Paper Competition at IGARSS 2003 in Toulouse, France. During the fall of 2006, she was an Advanced Study Program Graduate Visitor in the Earth Observing Laboratory of the National Center for Atmospheric Research (NCAR), Boulder, CO. At NCAR, her Ph.D. research focused on developing algorithms for the tomographic imaging of tropospheric water vapor using a ground-based network of compact microwave radiometers. 